POS PROCEEDINGS

\title{
CHARM 2016: Theory Summary
}

\section{Svjetlana Fajfer *}

Department of Physics, University of Ljubljana, Jadranska 19, 1000 Ljubljana, Slovenia and J. Stefan Institute, Jamova 39, P. O. Box 3000, 1001 Ljubljana, Slovenia E-mail: svjetlana.fajfer@ijs.si

A short summary of theoretical talks at CHARM 2016 is presented.

VIII International Workshop On Charm Physics

5-9 September, 2016

Bologna, Italy

${ }^{*}$ Speaker. 


\section{Introduction}

Charm quark being not light enough and not heavy enough is very interesting fundamental fermion of the Standard Model (SM). It plays very important role in studies of all SM gauge interactions. For example, within QCD in explaining structures of many new exotic states discovered at B-factories and BES III using lattice QCD tools, the charm quark has a special position. Many properties of charm mesons have to be very precisely known in order to constrain fundamental parameters of the SM. That motivates many Lattice QCD studies. In the electroweak sector the charm quark is very important. Long standing expectation of new physics discovery at low energies is not realised yet, although there are a few puzzles in B meson decays. Few years ago a possibility of $\mathrm{CP}$ violation occurring in two body non-leptonic charm meson decays initiated many analyses. Although, the $\mathrm{CP}$ violating asymmetry decreased from the first announcement, the search for direct in indirect effects of $\mathrm{CP}$ violation in still very puzzling. In Sec. 2 I briefly review recent results of QCD studies in charm systems as: spectroscopic properties, masses and structure of exotics. Sect. 3 is devoted to charm weak decays. Section 3 reviews the D-meson mixing. Sec. 4 contains the summary of flavour changing neutral current processes in charm sector. Sec. 5 summarises results on non-leptonic week decays and CP violation, while Sec. 6 contains the short report on multi-body charm decays.

\section{QCD in charm systems}

This section contains short review of the charm quark mass, charm contribution in heavy ion physics, charm spectroscopy.

\subsection{Charm quark mass}

The charm quark mass enters as a basic SM parameters in many theoretical studies. The lattice QCD has determined rather precisely the charm quark mass using different actions, with the three or four active flavours, different renormalization procedures and methods. The most recent value has been reported in Ref. [1]:

$$
\bar{m}_{c}\left(\bar{m}_{c}\right)=1.267(11) \mathrm{GeV} .
$$

As mentioned by the author of Ref. [2], the knowledge of Yukawa coupling is one of primary goals of the SM and hopefully in the further charm conferences this issue will be addressed after more data from LHC will be available. This will give us one of the crucial tests of the SM.

\subsection{Charm and heavy ions}

On CHARM 2016 a number of talks on charm contributions in heavy ions was presented: [3] [4], [5], [6], [7]. The matter produced in heavy-ion collisions is approached by the QCD and characterised by the phase diagrams [3]. Depending on the temperature and baryon density, as active coloured degrees of freedom in their descriptions enter quarks, gluons or hadrons. At the highest energies, nuclear collisions enables one to study transitions from a plasma of deconfined quarks and gluons to a hadronic gas and resonances in the region of high temperature, where baryon density almost vanishes. Such a regime is present during the universe thermal evolution. Following 
work of Ref. [3] transport calculations and experimental data indicate that heavy quarks strongly interact with the hot deconfined medium formed in high-energy nuclear collisions. Heavy-quark production is a short distance process due to the large quark mass. As a theoretical tool [3], the fixed order next-to-leading log calculation is developed for both regimes, low and high $p_{T}$. The data for $\mathrm{D}$ production at Alice are in good agreement with the above mentioned theoretical approach as well as POWHEG-BOX predictions.

\subsection{Charm production}

The charm production can be approached by the perturbative QCD tools. In particular, the production of charmed baryons and mesons in antiproton-proton annihilation was considered in Ref. [8]. In Ref. [9] theoretical aspects of the open charm production and spectroscopy were analysed. In the talk by Siddakov [10] a novel mechanism of the hidden-charm production in proton-nuclear collisions is considered. The authors found that the estimated cross-section exceeds the total cross-section via weak decays of $\Lambda_{b}$ in the decay in which penta-quarks are observed. Studies of the rapidity and transverse momentum distributions of penta-quarks might be useful in determining the $c \bar{c}$ component of this exotic baryon.

\subsection{Spectroscopy, exotics}

The theoretical analyses aimed to understand bound-states containing charm quarks in Fig.1, as given in Ref. [12,13] are numerous. There are many results presented at this conference: [13], [14], [15], [16], [17], [18], [19], [11], [20], [21]. Due to the relative heaviness of the charm quark mass, the non relativistic quark models were considered. In Ref. [14] the structure of the X, Y, Z mesons and other multi-quark states, like exotics and dibaryons is studied within the framework of a standard nonrelativistic quark-model picture. Conditions required for the existence of multiquark bound states or resonances contributing to the heavy hadron spectra are found and the role played by the entangled hadron - hadron thresholds is discussed. The experimental results on the penta-quark structure of $P_{c}^{+}$(4380) and $P_{c}^{+}$(4450) found by [22], have motivated study of [18] in which the constituent quark model has been able to explain the properties of the charmonium states from $J / \Psi$ to $X(3872)$. In this year new states $X(4140), X(4274), X(4500)$ and $X(4700)$ were found by the LHCb collaboration in the invariant $J / \Psi$ mass spectrum of the $\Lambda_{b}^{0} \rightarrow J / \Psi K^{-} p$ process [23, 24]. The authors of [18] were able to reconstruct $P_{c}^{+}(4380)$ and $P_{c}^{+}(4450)$ as the penta-quark states, while among the $X$ states, $X(4140)$ was found to be a cusp due to the presence of $D_{s} D_{s}^{*}$ states. In Ref. [15] a comparison of two nonrelativistic quark model models is done in order to test the description of the $X(3915)$ state. The one is the Cornell potential model, while the other one is the "unconventional" generalised screened potential. The author found that the decay $X(3915) \rightarrow \omega J / \Psi$ favours an unconventional description.

The production of charmed baryons and mesons in proton-antiproton annihilation was considered in Ref. [21]. It was found that the predicted production cross-sections of charmed baryons are typically in the order of $1 \mu \mathrm{b}$. In the case of charmed mesons the cross-sections can be in the range of 10-100 $\mu \mathrm{b}$. The lattice QCD made tremendous progress in the treatment of exotic bound states of quarks. The description of mesons scattering on the lattice became possible in the last few years (see e.g. [25]). In this workshop, Moir presented results of the Hadron Spectrum Collaboration on the coupled-channel $D \pi, D \eta, D_{s} \bar{K}$ in their scattering. In their study three poles were identified 
in the coupled channels of the $D \pi, D \eta, D_{s} \bar{K}$ modes. The $D_{0}^{*}(2400)$ state was found just below the $D \pi$ threshold. The collaboration observed a single S-wave bound-state pole in the elastic $D K$ scattering. Fast improvemetns of the lattice techniques might help in the future to fully understand the nature of charmonium spectrum and the content of many exotics. Additional theoretical tool, based on QCD in the description of charmonium states was presented by Brambilla [20]. The effective field theory with the multi-scales has been presented. The potential has weakly coupled perturbative non relativistic QCD (pNRQCD), as well as strongly coupled pNRQCD part, which can describe very well charmonium and bottomomium spectra.

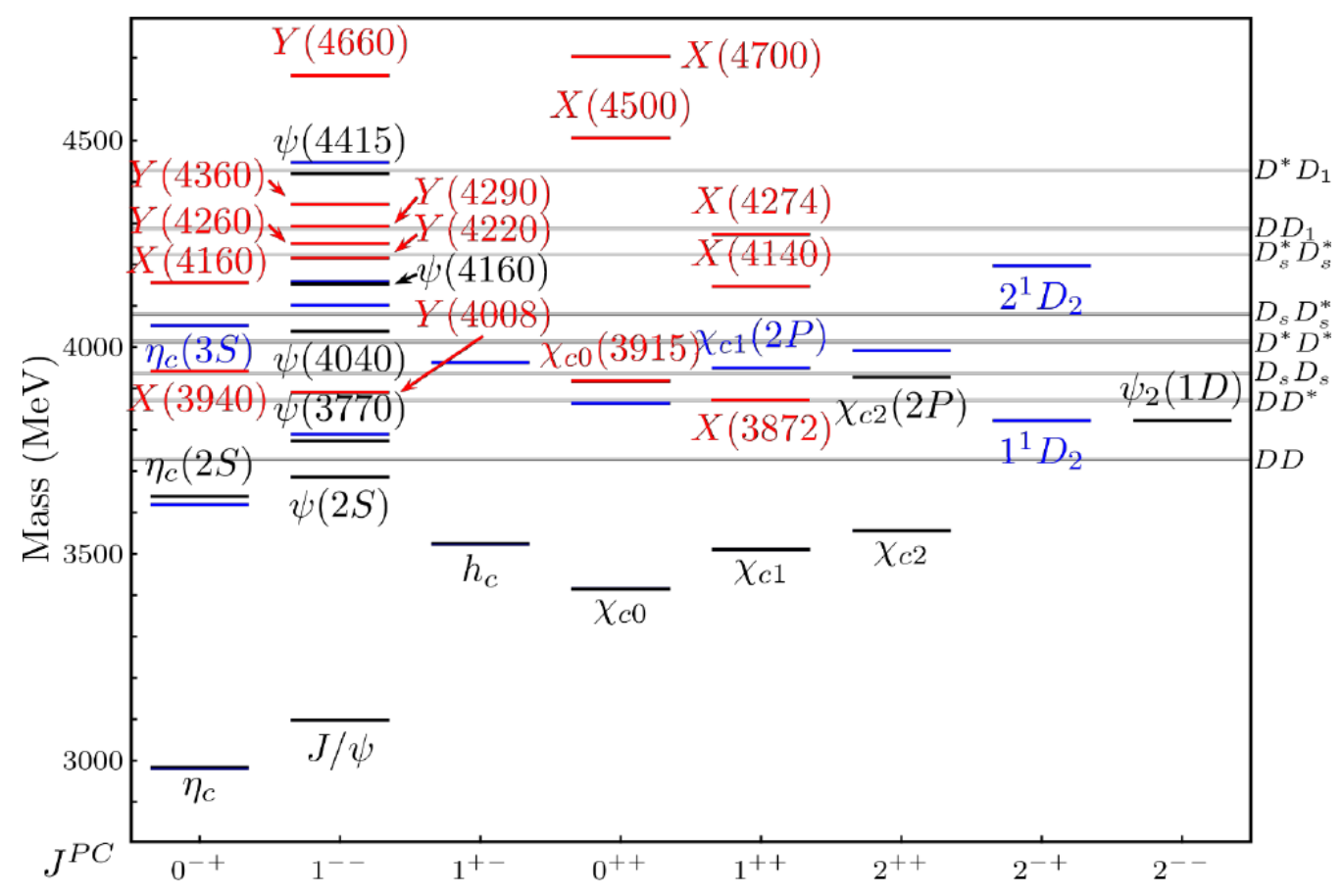

Figure 1: Exotic landscape.

\section{Charm leptonic and semileptonic decays}

In order to achieve high precision knowledge of all fundamental parameters of the SM, charm weak decays are very important. In addition to very precise measurements of the relevant observables, for many years we are following rapid progress in the knowledge of hadronic entries in the determination of the CKM matrix elements $V_{c d}, V_{c s}$ as given in the talks [26, 28, 27]. The $D$ and $D_{s}$ meson decay constants are now very well determined by the lattice QCD [26] $f_{D}=(212.15 \pm 1.45)$ $\mathrm{MeV}$ and $f_{D_{s}}=(248.83 \pm 1.27) \mathrm{MeV}$ with the errors of the order $1 \%$. According to the talk of El Khadra [26] the knowledge of form factors entering in the semileptonic decay amplitudes $D \rightarrow K / \pi l v_{l}$ has improved significantly. There are many studies with the three different values of the lattice spacing: $0.06 \mathrm{fm}-0.09 \mathrm{fm}$, different volumes: $2 \mathrm{fm}-3 \mathrm{fm}$ and with the pion mass in the 
range of $210 \mathrm{MeV}-440 \mathrm{MeV}$. The values of $f_{+}(0)$ for $D \rightarrow K / \pi l v_{l}$ are presented in Fig. 2 (see Ref. [26]). The small lattice errors decrease the errors on $\left|V_{c d}\right|,\left|V_{c s}\right|$ implying that the dominant uncertainty comes from the experimental side [26, 27]. In Fig 3. the results of FLAG2016 are shown [29]. In flavour physics nowadays B physics plays important role due to the few puzzles (see e.g.
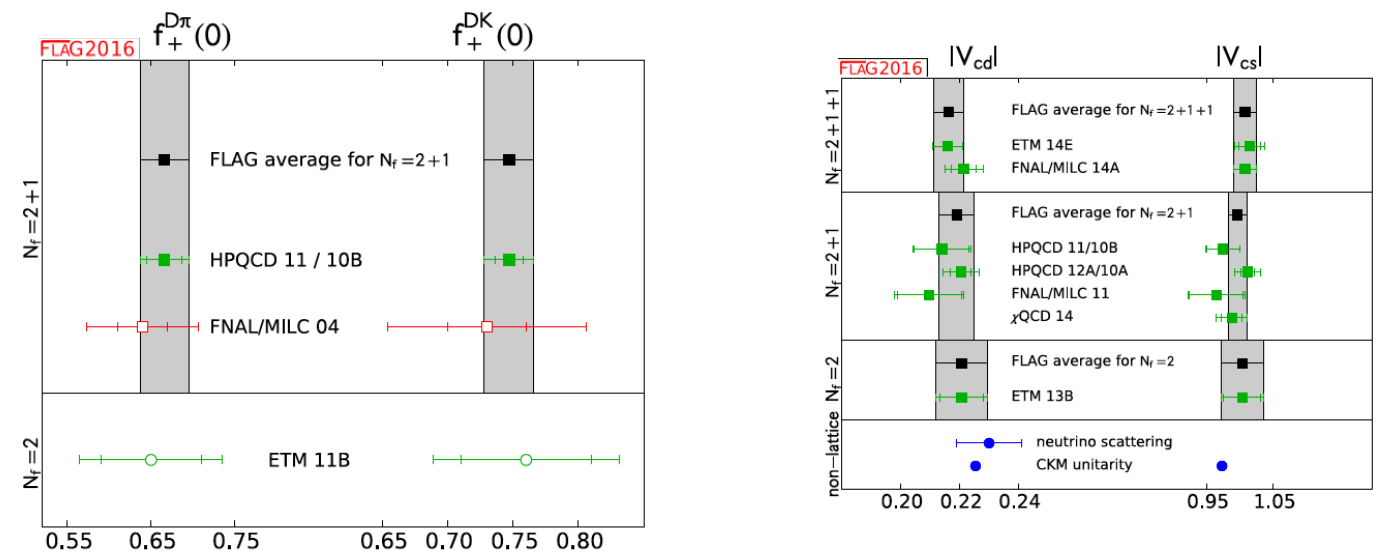

Figure 2: Left: FLAG averaging of the $\mid V_{c d}$ and $\left|V_{c s}\right|$ CKM matrix elements. Right: the form-factor $f_{+}(0)$ at $q^{2}=0$ for $D \rightarrow \pi / K l v_{l}$ decays.

[30]). Numerous studies question the existence of New Physics responsible for the deviation of experimental results from the SM predictions on the level of 3-4 standard deviations. It was pointed out by Martinelli [31], that flavour physics is so sensitive on NP due to the absence of flavour changing neutral currents at almost no $\mathrm{CP}$ violation at the tree level. That makes flavour physics very good testing ground of New Physics, competitive with electroweak precision measurements. The effects of NP in charm leptonic and semileptonic decays were discussed in talks of [27, 32]. In Ref. [27] the effective Lagrangian approach was used in which the SM was simply extended by the scalar or pseudoscalar operators. The leptonic decays $D_{s} \rightarrow l v_{l}$ and $D \rightarrow K^{*} l v_{l}$ might probe pseudo-scalar Wilson coefficient, while the decay $D \rightarrow K l v_{l}$ is a good probe of the scalar operator. The most restricting process is leptonic decays, due to the well known meson coupling constants. Naive estimate of the scale of New Physics can be obtained from the bound on the pseudo-scalar Wilson coefficient. It was estimated that the scale of NP should be larger than $1.7 \mathrm{TeV}$ [27]. A number of ultraviolet complete theories can give contributions to both operators, e.g. two Higgs doublet model, new charged current or leptoquark exchange.

\section{FCNC processes}

There are two kinds of FCNC transitions: one in which the charm changes for one unit (rare charm decays) and the processes with the charm change by the two units ( $D-\bar{D}$ oscillations). As mentioned in the presentation of [28], these transitions are very useful in constraining New Physics contributions. Both transitions are now the only low-energy probe of up-quarks flavour changing neutral currents (FCNCs). 

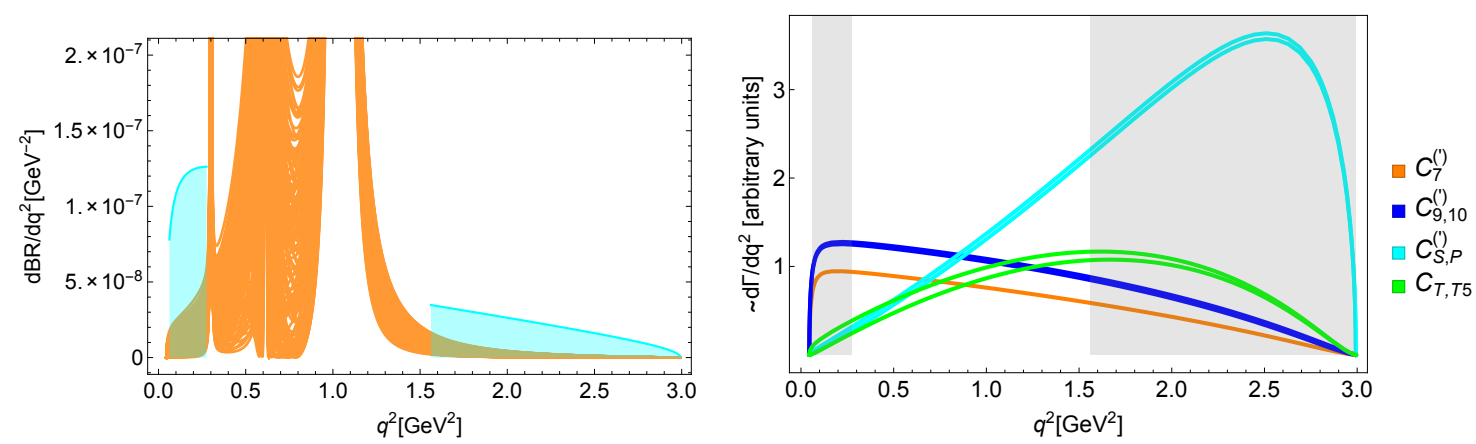

Figure 3: $\mathrm{SM}$ resonant contributions in $D^{+} \rightarrow \pi^{+} \mu^{+} \mu^{-}$shown in orange. Cyan regions correspond to a scenario with constant decay amplitude that would saturate LHCb bounds (left figure). Comparison of short distance spectrum sensitivities to different Wilson coefficients. Grey regions indicate the LHCb experimental low- and high- $q^{2}$ bins (right figure).

\subsection{Rare charm decays}

In the talk of [26] it was mentioned that in rare charm decays in which $c \rightarrow u l^{+} l^{-}$transition occurs, long distance contributions are dominant (see recent studies [33, 34]). The relevant effective Hamiltonian at scale $\mu \sim m_{c}$ can be written as:

$$
\mathscr{H}_{\text {eff }}=\lambda_{d} \mathscr{H}^{d}+\lambda_{s} \mathscr{H}^{s}+\lambda_{b} \mathscr{H}^{\text {peng }}
$$

where each of them is weighted by an appropriate combination $\lambda_{q}=V_{u q} V_{c q}^{*}$ of Cabibbo-KobayashiMaskawa (CKM) matrix elements. Virtual contributions of states heavier than charm quark is by convention contained within:

$$
\mathscr{H}^{\text {peng }}=-\frac{4 G_{F}}{\sqrt{2}} \sum_{i=3, \ldots, 10} C_{i} \mathscr{O}_{i}
$$

The operators appearing in the above Hamiltonian are defined in [34]. Motivated by the improvements of the bounds on rare charm decays by the LHCb experiment as given in [34], the bounds on the effective Wilson coefficients were determined. The upper bounds on the effective Wilson coefficients are given in Fig. 2 (left). The strongest constraints on $C_{10}, C_{P}, C_{S}$ and $C_{10}^{\prime}, C_{P}^{\prime}, C_{S}^{\prime}$ are obtained from the bound on the branching fraction of the $D^{0} \rightarrow \mu^{+} \mu^{-}$decay. The non-resonant differential decay width distribution gives bounds on $C_{i}, i=7,9,10, S, P, T, T 5$ as well as on the coefficients of the operators of opposite chirality. The differential branching ratio at the high dilepton invariant mass bin is more constraining than the one at the low di-lepton invariant mass bin. This statement applies in particular to the contributions of the scalar and pseudo-scalar operators. It was found in [34] that forward-backward asymmetry is sensitive to the combination of scalar and tensor coefficients at high- $q^{2}$.

\subsection{Neutral charm meson mixing}

The $D^{0}-\bar{D}^{0}$ mixing amplitude receives contributions of the short and long distance dynamics $[2,26]$, as presented in Fig. 4 [26]. The short distance contribution suffers from the strong GIM cancellation due to down-quarks contributions in the box diagrams. Unfortunately, the long 
distance contribution is difficult to determine, since intermediate states includes multiple hadrons. The lattice QCD (see [26]) can approach the short distance contribution relying on the same methods as for B meson mixing. This calculations are particularly important, since NP might contribute to the transition amplitude. For the long distance calculation formalism for multi-hadron states is still under development [35]. The effective Hamiltonian describing short distance dynamics has 5 operators (see [26]), which matrix elements $<D^{0}\left|\mathscr{O}_{i}\right| \bar{D}^{0}>(\mu)=e_{i} M_{D}^{2} f_{D}^{2} B_{D}^{(i)}(\mu)$ introduce the socalled bag parameters $B_{D}^{(i)}$. In Fig. 5 (from [26]) the summary of lattice results on bag parameters are given. On the other hand the existing data on the $\mathrm{D}$-mixing might put a lower bound on the

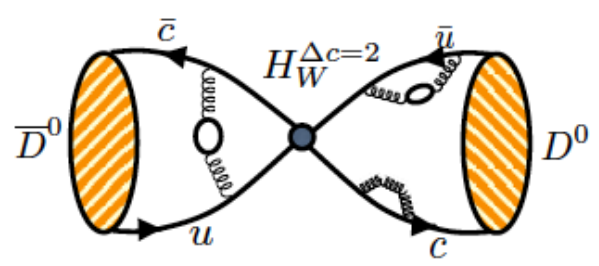

short distance

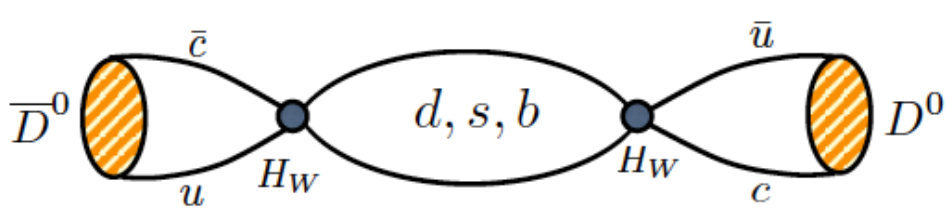

long distance

Figure 4: Short and long distance contribution in D meson mixing

scale of New Physics as discussed in [36]. The scale of NP can be obtained from:

$$
\Lambda=\sqrt{\frac{L F_{i}}{C_{i}(\Lambda)}} .
$$

The factor $L$ comes from one loop contribution of NP and $F_{i}$ is a number of flavours in the theory, while $C_{i}(\Lambda)$ ia the Wilson coefficient at scale $\Lambda$. By assuming that $L=1$ and $F_{i}=1$ one derives lower bound from $\mathscr{O}_{i}$ operators $\Lambda=3.6 \times 10^{4} \mathrm{TeV}$. Hopefully, further developments of new methods for the calculation of long distance contributions to the D-mixing amplitude, by the lattice QCD would help in our knowledge of FCNC processes. 


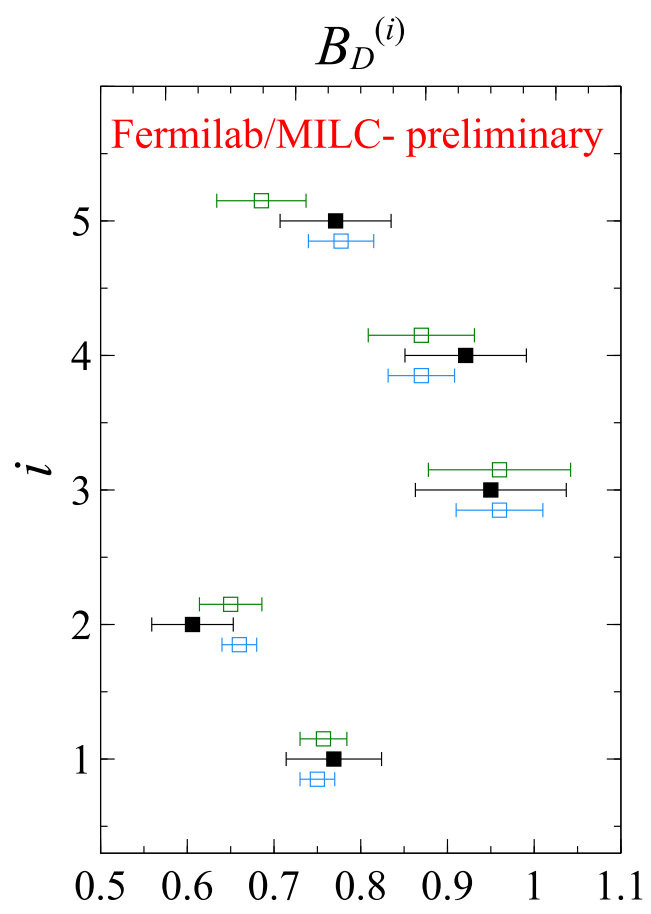

Figure 5: The bag parameters calculated by ETM collaboration with number degrees of freedom $n_{f}=$ $2+1+1$ (green), by FERMILAB/MILC with $n_{f}=2+1$ (black) and by ETM with $n_{f}=2$ by ETM (blue) .

\section{Nonleptonic week decays and $\mathrm{CP}$ violation}

Both topics, nonleptonic week decays and CP violation, were addressed in Ref. [37]. The speaker Pietro Santorelli reviewed recent analysis based on the $S U_{F}(3)$ breaking [37]. The amplitude for $D \rightarrow P P$ can be reduced on the three reduced matrix elements by using the Wigner-Eckart theorem. The number of parameters varies and depends weather the $S U_{F}(3)$ breaking is accounted or not, as well as weather CP violating asymmetries are included into the consideration $[38,39]$. The global fit to the two body charm decay amplitudes, based on $S U_{F}(3)$ and including final state interactions have been performed long time ago by the authors of [40]. Regarding the direct CP violation, based on the $S_{F}(3)$ study, it was found that it is not possible to predict CP asymmetry, but one can construct combinations of $\mathrm{CP}$ asymmetries containing only those topological amplitudes obtained by the fit [41]. An interesting result of this topological study was offered in [42] predicting that for the $D^{0} \rightarrow K_{S} K_{S}$ decay direct CP asymmetry is $\leq 1.1 \%$.

\section{Multi-body charm decays}

In the study of multi-body $\mathrm{D}$ decays there was a number of contributions at this conference [43, 44, 45, 46, 47]. The main reason why to investigate such processes are following: In Quantum Mechanics the wave function $\Psi$ is the fundamental object which is not measurable, while $|\Psi|^{2}$ is 
a measurable. In order to obtain a complete information on the wave function in order to study CP violation, one needs to know phases entering in the wave function. In Ref. [47] it was pointed

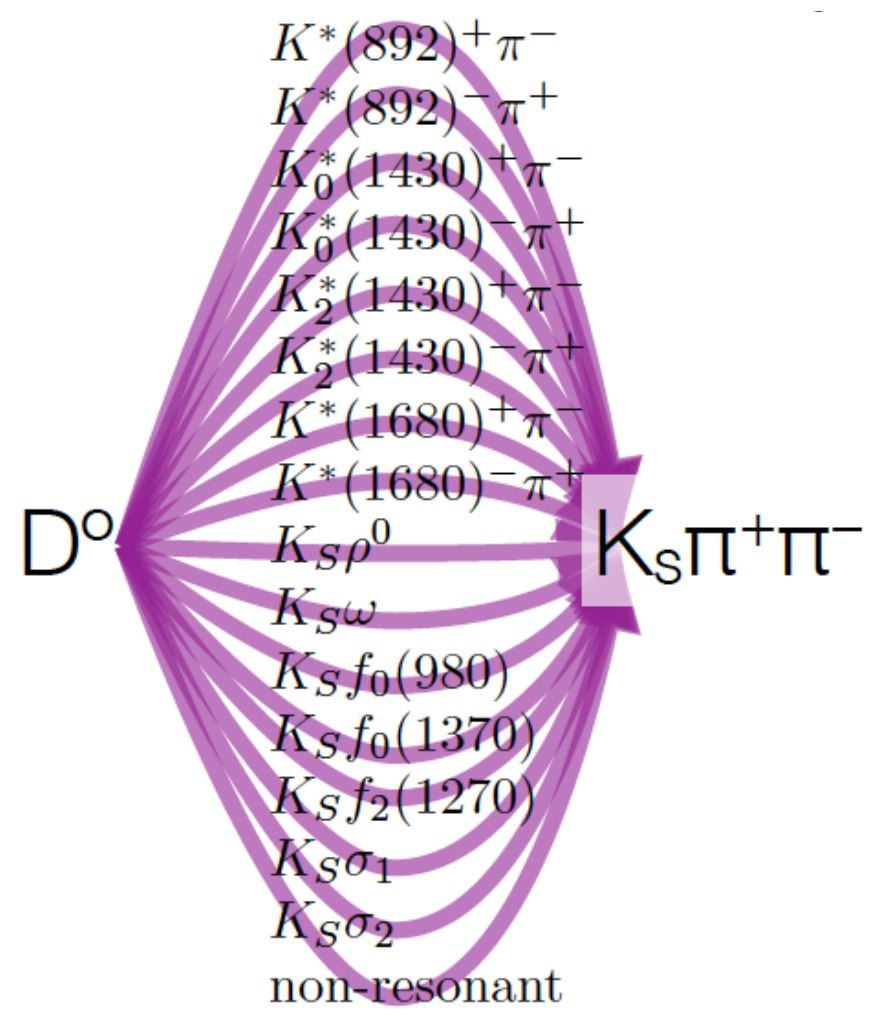

Figure 6: Intermediate states in the decay $D \rightarrow K_{s} \pi^{+} \pi^{-}$.

out that charm inputs are of great importance to the extraction of the gamma angle of the unitary triangle and therefore, also to the analysis of NP effects. In Re. [43] multi-channels were considered in $D \rightarrow K_{s} \pi^{+} \pi^{-}$decay (see Fig. 6) and it was found how this "mixed" approach uses the "Breit-Wigner narrowish resonances and uses those as an interferometer for a model independent methods to parametrise broad (typically S-wave) overlapping resonances". In the talk by Nakamura [44] the coupled-channel Dalitz-plot analyses study of $D^{+} \rightarrow K^{-} \pi^{+} \pi^{+}$decay was done and large rescattering effects were found.

\section{Concluding remarks}

I briefly report on the main results presented at CHARM 2016. It is very impressive progress since CRARM 2015 conference. In such a short period of time many new results were obtained starting with the more precise knowledge of SM fundamental parameters, spectroscopic properties, weak decay dynamics, as well as questioning the NP presence.

\section{ACKNOWLEDGEMENTS}

I acknowledge support of the Slovenian Research Agency. I am indebted to A. Pilloni, A. El-Khadra and J. Rademacker for granting permission to use their plots in this talk. 


\section{References}

[1] Y. Maezawa and P. Petreczky, Phys. Rev. D 94 (2016) no.3, 034507 doi:10.1103/PhysRevD.94.034507 [arXiv:1606.08798 [hep-lat]].

[2] A. Lenz, arXiv:1610.07943 [hep-ph].

[3] A. Beraudo, arXiv:1610.08269 [hep-ph].

[4] Frank Geurts, these proceedings; talk at CHARM 2016.

[5] Francois Arleo, these proceedings; talk at CHARM 2016.

[6] Antonio Vairo, these proceedings; talk at CHARM 2016.

[7] Martin Cleven, these proceedings; talk at CHARM 2016.

[8] Johann Haidenbauer, these proceedings; talk at CHARM 2016.

[9] Wei Wang, these proceedings; talk at CHARM 2016.

[10] Marat Siddikov, these proceedings; talk at CHARM 2016.

[11] Sinead Ryan, these proceedings; talk at CHARM 2016.

[12] A. Esposito, A. L. Guerrieri, F. Piccinini, A. Pilloni and A. D. Polosa, Int. J. Mod. Phys. A 30 (2015) 1530002 doi:10.1142/S0217751X15300021 [arXiv:1411.5997 [hep-ph]].

[13] Alessandro Pilloni, these proceedings; talk at CHARM 2016.

[14] Alfredo Valcarce, these proceedings; talk at CHARM 2016.

[15] Pedro Gonzalez, these proceedings; talk at CHARM 2016.

[16] Graham Moir, these proceedings; talk at CHARM 2016.

[17] Timothy Burns, these proceedings; talk at CHARM 2016.

[18] Francisco Fernandez, these proceedings; talk at CHARM 2016.

[19] Gavin Cheung, these proceedings; talk at CHARM 2016.

[20] Nora Brambilla, these proceedings; talk at CHARM 2016.

[21] Johann Haidenbauer, these proceedings; talk at CHARM 2016.

[22] R. Aaij et al. [LHCb Collaboration], Phys. Rev. Lett. 115 (2015) 072001 doi:10.1103/PhysRevLett.115.072001 [arXiv:1507.03414 [hep-ex]].

[23] R. Aaij et al. [LHCb Collaboration], Phys. Rev. Lett. 117 (2016) no.8, 082002 doi:10.1103/PhysRevLett.117.082002 [arXiv:1604.05708 [hep-ex]].

[24] R. Aaij et al. [LHCb Collaboration], Phys. Rev. Lett. 117 (2016) no.8, 082003 Addendum: [Phys. Rev. Lett. 117 (2016) no.10, 109902] doi:10.1103/PhysRevLett.117.082003, 10.1103/PhysRevLett.117.109902 [arXiv:1606.06999 [hep-ex]].

[25] S. Prelovsek, arXiv:1508.07322 [hep-lat].

[26] Aida El-Khadra, these proceedings; talk at CHARM 2016.

[27] Nejc Kosnik, these proceedings; talk at CHARM 2016.

[28] Lorenza Riggio, these proceedings; talk at CHARM 2016. 
[29] S. Aoki et al., doi:10.3204/PUBDB-2016-03637 arXiv:1607.00299 [hep-lat].

[30] A. Crivellin, PoS BEAUTY 2016 (2016) 042 [arXiv:1606.06861 [hep-ph]].

[31] Guido Martinelli, these proceedings; talk at CHARM 2016.

[32] Ayan Paul, these proceedings; talk at CHARM 2016.

[33] S. de Boer and G. Hiller, Phys. Rev. D 93 (2016) no.7, 074001 doi:10.1103/PhysRevD.93.074001 [arXiv:1510.00311 [hep-ph]].

[34] S. Fajfer and N. Koĺnik, Eur. Phys. J. C 75 (2015) no.12, 567 doi:10.1140/epjc/s10052-015-3801-2 [arXiv:1510.00965 [hep-ph]].

[35] M. T. Hansen and S. R. Sharpe, Phys. Rev. D 93 (2016) no.9, 096006 doi:10.1103/PhysRevD.93.096006 [arXiv:1602.00324 [hep-lat]].

[36] Marco Ciuchini, these proceedings; talk at CHARM 2016.

[37] Pietro Santorelli, these proceedings; talk at CHARM 2016.

[38] G. Hiller, M. Jung and S. Schacht, Phys. Rev. D 87 (2013) no.1, 014024 doi:10.1103/PhysRevD.87.014024 [arXiv:1211.3734 [hep-ph]].

[39] S. Müller, U. Nierste and S. Schacht, Phys. Rev. D 92 (2015) no.1, 014004 doi:10.1103/PhysRevD.92.014004 [arXiv:1503.06759 [hep-ph]].

[40] F. Buccella, M. Lusignoli, G. Miele, A. Pugliese and P. Santorelli, Phys. Rev. D 51 (1995) 3478 doi:10.1103/PhysRevD.51.3478 [hep-ph/9411286].

[41] S. Müller, U. Nierste and S. Schacht, Phys. Rev. Lett. 115 (2015) no.25, 251802 doi:10.1103/PhysRevLett.115.251802 [arXiv:1506.04121 [hep-ph]].

[42] U. Nierste and S. Schacht, Phys. Rev. D 92 (2015) no.5, 054036 doi:10.1103/PhysRevD.92.054036 [arXiv:1508.00074 [hep-ph]].

[43] Jonas Rademacker, these proceedings; talk at CHARM 2016.

[44] Benoit Loiseau, these proceedings; talk at CHARM 2016.

[45] Satoshi Nakamura, these proceedings; talk at CHARM 2016.

[46] Patricia Magalhaes, these proceedings; talk at CHARM 2016.

[47] Denis Derkach, these proceedings; talk at CHARM 2016. 